\title{
Clinical Value of Combined Determination of Serum B7-H4 with Carcinoembryonic Antigen, Osteopontin, or Tissue Polypeptide- Specific Antigen for the Diagnosis of Colorectal Cancer
}

\author{
Peng Wang, ${ }^{1,2}$ Chun Li, ${ }^{1,2}$ Fan Zhang, ${ }^{1}$ Xuzhe Ma, ${ }^{1,2}$ and Xiaodong Gai $\mathbb{D}^{1,2}$ \\ ${ }^{1}$ Department of Pathology, Medical School of Beihua University, Jilin, China \\ ${ }^{2}$ Key Laboratory of Molecular Medicine, Jilin Province Education Department, Beihua University, Jilin, China \\ Correspondence should be addressed to Xiaodong Gai; bhdxgaixiaodong@126.com
}

Received 29 May 2018; Accepted 7 August 2018; Published 27 September 2018

Academic Editor: Upender Manne

Copyright (c) 2018 Peng Wang et al. This is an open access article distributed under the Creative Commons Attribution License, which permits unrestricted use, distribution, and reproduction in any medium, provided the original work is properly cited.

\begin{abstract}
Aim. B7-H4 is member of the B7 family that negatively regulates the immune response, which are associated with tumor development and prognosis. The present study is aimed at examining serum B7-H4 expression and exploring its contribution to diagnosis in patients with colorectal cancer. Methods. We determined serum expressions of B7-H4, carcinoembryonic antigen (CEA), osteopontin (OPN), and tissue polypeptide-specific antigen (TPS) in 59 patients with colorectal cancer and 29 healthy volunteers and analyzed the diagnostic value of B7-H4 combined with CEA, OPN, or TPS detection for colorectal cancer. B7-H4, OPN, and TPS serum expressions were measured by enzyme-linked immunosorbent assay, and CEA was measured by electrochemical luminescence detection. Results. Serum B7-H4 levels were significantly higher in colorectal cancer patients compared with paired normal controls $(P=0.001)$. B7-H4 serum level was positively correlated with infiltration depth, tumor masses, and lymph node metastasis $(P=0.004, P=0.016$, and $P=0.0052$, respectively). We also detected serum expression of $\mathrm{B} 7-\mathrm{H} 4$ before and after radical resection and showed that $\mathrm{B} 7-\mathrm{H} 4$ levels decreased significantly during the first week postoperation $(P=0.0064)$. We used receiver operating characteristic $(\mathrm{ROC})$ curve analysis to indicate the potential diagnostic values of these markers. The areas under the ROC curves (AUC) for B7-H4, OPN, TPS, and CEA were $0.867,0.805,0.812$, and 0.833 , respectively. The optimal sensitivity and specificity of B7-H4 for discriminating between colon cancer patients and healthy controls were $88.2 \%$ and $86.7 \%$, respectively, using a cut-off of value of $78.89 \mathrm{ng} / \mathrm{mL}$. However, combined ROC analysis using B7-H4 and CEA revealed an AUC of 0.929, with a sensitivity of $98.9 \%$ and a specificity of $80.4 \%$ for discriminating colon cancer patients from healthy controls. Conclusions. B7-H4 was highly expressed in the serum in colorectal cancer patients. Detection of B7-H4 plus CEA showed significantly increased sensitivity and specificity for discriminating between colorectal cancer patients and healthy controls compared to individual detection of these markers. Combined detection of serum B7-H4 and CEA may thus have the potential to become a new laboratory method for the early clinical diagnosis and prognostic evaluation of colorectal cancer.
\end{abstract}

\section{Introduction}

Colorectal cancer $(\mathrm{CRC})$ is one of the most frequent cancers diagnosed in high-income countries and most middleincome countries [1], accounting for nearly $8.5 \%$ of total cancer-related death annually [2, 3]. Moreover, many patients with CRC are not diagnosed until the disease is in its advanced stages and is no longer treatable. Extensive research into the factors related to CRC suggests a crucial role of early diagnosis, though several studies have failed to prove a causal relationship [1]. Tumor initiation and development have been shown to be complex processes involving multiple stages and regulation of multiple genes. Although CRC develops as a consequence of local tissue changes [4, 5], methods of predicting cancer risk before the appearance of morphological changes in the colon remain unclear.

Successful detection of CRC in the early stages is crucial for providing effective therapies for this aggressive and malignant disease, and screening for sensitive and specific tissue or body-fluid biomarkers that may provide an accurate 
early diagnosis of CRC has been an important task for both clinicians and cancer biologists in recent decades. Although carcinoembryonic antigen (CEA) is currently the most commonly used serum tumor marker for CRC [6-9], it is not recommended as a screening or diagnostic tool for this neoplasm, especially in the early stages. B7-H4 is a B7 family molecule and a highly evolutionarily conserved transmembrane protein that shares approximately $25 \%$ amino acid homology in the extracellular portion with other B7 family members [10]. B7-H4 ligation of $\mathrm{T}$ cells causes cell cycle arrest and has a profound inhibitory effect on cell growth, cytokine secretion, and the development of cytotoxicity [11]. Administration of B7-H4 Ig to mice impaired antigenspecific T-cell responses, whereas blockade of endogenous B7-H4 by specific monoclonal antibody promoted T-cell responses [12].

B7-H4 can regulate the T-cell immune response by inhibiting T-cell proliferation, cytokine production, and the cell cycle [13] and may thus participate in the negative regulation of cell-mediated immunity in normal peripheral tissues [12]. In contrast, $\mathrm{B} 7-\mathrm{H} 4$ has been shown to be overexpressed in some cancers, such as ovarian cancer, lung cancer, and breast cancer [14-16]. We previously demonstrated that B7-H4 had a strong prognostic significance and promoted tumor tolerance and might contribute to Treg development in the CRC tolerogenic milieu [17]. Higher serum B7-H4 levels have also been reported in CRC patients and were closely correlated with many important clinicopathological parameters, further supporting its potential value for the early diagnosis and prediction of disease prognosis.

In addition to $\mathrm{B} 7-\mathrm{H} 4$, numerous tumor markers have been found and used in clinical practice [18]. Tumor markers may reflect the presence and growth of the tumor, thus providing information on the nature of the tumor and potentially aiding its early diagnosis. However, the expression pattern and diagnostic value of $\mathrm{B} 7-\mathrm{H} 4$ protein have not yet been investigated. This study therefore investigated the serum expression levels of $\mathrm{B} 7-\mathrm{H} 4$ in patients with CRC. We also explored the diagnostic sensitivity and accuracy of B7-H4 in combination with serum osteopontin (OPN) [19], tissue polypeptide-specific antigen (TPS) [20], and CEA [21] for the early detection of CRC.

\section{Materials and Methods}

2.1. Patients and Serum Samples. This study was approved by the Research Ethics Committee of the General Hospital of Jilin Chemical Industry, China. Written informed consent was obtained from all the patients according to the committee's regulations. All samples were handled and anonymized according to the ethical and legal standards. Fifty-nine paraffin-embedded CRC samples and matched serum samples were provided by the General Hospital of Jilin Chemical Industry for patients with complete histopathology and follow-up information from 2013 to 2014. None of the patients received preoperative chemotherapy or radiotherapy. The clinicopathological characteristics of the patients are detailed in Table 1.
TABLE 1: Clinical material of patient and control groups.

\begin{tabular}{|c|c|c|c|}
\hline Clinical features & Patient group & Control group & $\begin{array}{l}\text { Percentage } \\
\text { (\% of total) }\end{array}$ \\
\hline \multicolumn{4}{|l|}{ Gender } \\
\hline Male & 41 & 14 & $69.49 / 58.33$ \\
\hline Female & 18 & 10 & $30.51 / 41.67$ \\
\hline \multicolumn{4}{|l|}{ Age } \\
\hline$<60$ & 32 & 14 & $54.37 / 58.33$ \\
\hline$\geq 60$ & 27 & 10 & $45.63 / 41.67$ \\
\hline \multicolumn{4}{|l|}{ T stage } \\
\hline $\mathrm{T} 1$ & 0 & & 0.00 \\
\hline $\mathrm{T} 2$ & 14 & & 23.73 \\
\hline $\mathrm{T} 3$ & 21 & & 35.59 \\
\hline $\mathrm{T} 4$ & 24 & & 40.68 \\
\hline \multicolumn{4}{|l|}{$\mathrm{N}$ stage } \\
\hline N0 & 6 & & 10.17 \\
\hline N1 & 11 & & 18.64 \\
\hline N2 & 42 & & 71.19 \\
\hline \multicolumn{4}{|l|}{ M stage } \\
\hline M0 & 48 & & 81.36 \\
\hline M1 & 11 & & 18.64 \\
\hline \multicolumn{4}{|l|}{ TNM stage } \\
\hline I & 2 & & 3.39 \\
\hline II & 4 & & 6.78 \\
\hline III & 42 & & 71.19 \\
\hline IV & 11 & & 18.64 \\
\hline \multicolumn{4}{|l|}{ Differentiation } \\
\hline Low & 7 & & 11.86 \\
\hline Middle & 47 & & 79.66 \\
\hline High & 5 & & 8.48 \\
\hline \multicolumn{4}{|l|}{ Histology } \\
\hline Squamous & 2 & & 3.39 \\
\hline Mucoid & 57 & & 96.61 \\
\hline
\end{tabular}

Note: $\mathrm{T}$ means the depth of tumor cell infiltration; $\mathrm{N}$ means the level of lymph node metastasis; $\mathrm{M}$ means the condition of metastasis.

Twenty-four healthy age- and sex-matched controls were recruited from among people who came for general health examinations. Blood samples were kept at room temperature for $22 \mathrm{~h}$, and serum was obtained by centrifugation at $3000 \times \mathrm{g}$ at $4^{\circ} \mathrm{C}$ for $15 \mathrm{~min}$. The serum was removed immediately and frozen on dry ice at $-80^{\circ} \mathrm{C}$ until use.

2.2. Measurement of Serum B7-H4, OPN, and TPS. B7-H4, OPN, and TPS serum levels were measured by enzymelinked immunosorbent assay (ELISA) methods, as described previously [22]. B7-H4 levels were also determined using an assay kit (Rapidbio, Hayward, CA, USA). The sensitivities of the B7-H4 immunoassays were $0.156 \mathrm{ng} / \mathrm{mL}$. Briefly, $100 \mu \mathrm{L}$ of serum was placed in each well of the ELISA plate and incubated for $45 \mathrm{~min}$ at $37^{\circ} \mathrm{C}$. The plates were washed four times with buffer and incubated with $50 \mu \mathrm{L}$ of detection antibody $(0.2 \mu \mathrm{g} / \mathrm{mL})$ at $37^{\circ} \mathrm{C}$ for $1 \mathrm{~h}$. After five washes, the plates were incubated with $100 \mu \mathrm{L}$ horseradish peroxidase 
TABLE 2: The comparison of serum concentrations of B7-H4/OPN/TPS/CEA between CRC patient group and control group.

\begin{tabular}{lccccc}
\hline Group & $n$ & B7-H4 & OPN & TPS & CEA \\
\hline CRC & 59 & $95.78 \pm 13.42$ & $116.14 \pm 56.31$ & $98.77 \pm 27.62$ & $77.17 \pm 81.09$ \\
Control & 24 & $63.20 \pm 15.69$ & $78.63 \pm 16.43$ & $76.40 \pm 15.04$ & $1.95 \pm 1.04$ \\
$t$ & & 9.539 & 2.720 & 3.738 & 2.332 \\
$P$ & & 0.001 & 0.032 & 0.018 & 0.008 \\
\hline
\end{tabular}

solution $(1: 5000)$ for $30 \mathrm{~min}$ at $37^{\circ} \mathrm{C}$ and then reacted with the enzyme substrate p-nitrophenyl phosphate (Cat N1891, Sigma-Aldrich, WI, USA) for $30 \mathrm{~min}$ at room temperature. The absorbance was then read at $450 \mathrm{~nm}$ using a microplate ELISA reader (Multiskan MK3, Thermo Scientific, MA, USA). A corresponding standard curve was generated using the provided standards and was used to calculate the quantities of B7-H4, OPN, and TPS in each serum sample.

2.3. Electrochemical Luminescence Detection of Serum CEA. Levels of CEA in the serum samples were measured using a two-site microtiter plate-based immunoassay with electrochemical luminescence detection (Cobas601 Kit, Roche, Germany) according to the manufacturer's instructions. At least three independent experiments were conducted for each case. The accuracy of the results for the quality control samples and sensitivity met the experimental requirements.

2.4. Statistical Analysis. Data are expressed as mean \pm standard deviation. Comparisons among multiple groups and between two groups were conducted using analysis of variance with Tukey's post hoc test and Student's $t$-test, respectively. Variables following a nonnormal distribution were logarithmically transformed (natural logarithm) before use in parametric analyses. Pearson correlation and $\chi^{2}$ test or Fisher's exact test were used for comparison and estimation of correlations between B7-H4, OPN, TPS, and CEA serum expressions and clinicopathological tumor parameters as infiltration, metastasized, lymph node metastasis, or differentiation. Specificity and sensitivity of serum B7-H4, OPN, TPS, and CEA expression levels for CRC patients with control of follow-up were evaluated with receiver operating characteristic (ROC) curve analysis. Diagnostic accuracy of biomarkers was also determined by obtaining the largest possible area under the curve (AUC) in ROC analysis. Bonferroni data were analyzed using SPSS v.23.0 software. For all comparisons, $P<0.05$ was considered statistically significant. Graphs were plotted using GraphPad Prism v.6.0 software (GraphPad Inc., La Jolla, CA, USA).

\section{Results}

3.1. Comparison of B7-H4, OPN, TPS, and CEA Levels between CRC Patients and Controls. We analyzed B7-H4, OPN, TPS, and CEA protein levels in 59 CRC and 29 corresponding normal serum samples. The mean B7-H4 serum level in CRC patients $(95.78 \pm 13.42 \mathrm{ng} / \mathrm{mL})$ was significantly higher than that in healthy controls $(63.20 \pm 15.69 \mathrm{ng} / \mathrm{mL})$ $(P=0.001)$. The mean OPN, TPS, and CEA serum expressions were also significantly higher in CRC samples compared with controls (OPN: $116.14 \pm 36.31$ vs. $78.69 \pm 16.45 \mathrm{ng} / \mathrm{mL}$, $P=0.032$; TPS: $98.77 \pm 27.62$ vs. $76.40 \pm 15.04 \mathrm{U} / \mathrm{L}, P=0.018$; CEA: $77.17 \pm 81.09$ vs. $1.95 \pm 1.04 \mathrm{ng} / \mathrm{mL}, P=0.008)$. Summarized data for all markers are shown in Table 2. There was no significant correlation between B7-H4, OPN, TPS, or CEA expression and age or gender (Table 3 ).

\subsection{Diagnostic Value by Receiver Operating Characteristic} Curve Analysis. The above results and previous reports $[17-19,22,23]$ indicated that serum expressions of B7-H4, OPN, TPS, and CEA were markedly higher in tumor patients than in healthy controls. We investigated the potential diagnostic roles of these markers in CRC by receiver operating characteristic (ROC) curve analysis and the area under curve (AUC). The cut-off values based on the ROC analysis were $78.89 \mathrm{ng} / \mathrm{mL}$ for $\mathrm{B} 7-\mathrm{H} 4,95.06 \mathrm{ng} / \mathrm{mL}$ for OPN, $91.44 \mathrm{U} / \mathrm{L}$ for TPS, and $3.4 \mathrm{ng} / \mathrm{L}$ for CEA. The ROC curve demonstrated optimal sensitivity (0.882) of B7-H4 serum levels for distinguishing between $\mathrm{CRC}$ patients and healthy controls at a threshold of $78.89 \mathrm{ng} / \mathrm{mL}$, with an AUC of 0.867 , while CEA levels showed the highest specificity (0.833) (Figure 1). Combined ROC curve analysis using B7-H4 and CEA revealed an AUC of 0.929 with a sensitivity of $98.9 \%$ and a specificity of $80.4 \%$ for discriminating CRC patients from healthy controls. (Figure 1 and Table 4). While the AUC of combinational B7-H4 and OPN is 0.887 with sensitivity of $99.2 \%$ and specificity of $55.9 \%$, ROC curve of combined B7-H4 and TPS revealed an AUC of 0.891 with a sensitivity of $99.0 \%$ and a specificity of $60.1 \%$. Among these biomarkers, the best accuracy (Youden's index) of diagnosis for CRC was B7-H4 and CEA (0.793) (Table 4). The results indicated that combined detection using $\mathrm{B} 7-\mathrm{H} 4$ and CEA significantly improved the sensitivity and specificity for diagnosing CRC (Figure 2).

3.3. Correlation between Serum Expressions of B7-H4, OPN, TPS, and CEA and Clinicopathological Parameters of CRC. The correlations between serum B7-H4, OPN, TPS, and CEA protein levels and clinicopathologic features are summarized in Table 3. There was no significant correlation between TPS $(P=0.962)$ or CEA $(P=0.365)$ expression and tumor infiltration. However, high serum expressions of B7-H4 $(P=0.0001)$ and OPN $(P=0.040)$ were significantly correlated with tumor infiltration. B7-H4 $(P=0.016)$ and TPS $(P=0.027)$ levels were also significantly correlated with tumor masses, while OPN $(P=0.567)$ and CEA $(P=0.253)$ levels were not related to CRC tumor masses (Table 3). B7-H4 serum expression was significantly higher in CRC patients with more lymph node metastases $(\geq 4)(110.70 \pm$ $16.52 \mathrm{ng} / \mathrm{mL}$ ) compared with patients with fewer lymph 
TABLE 3: The association between serum B7-H4, OPN, TPS, and CEA levels and CRC clinicopathological parameters.

\begin{tabular}{|c|c|c|c|c|c|c|c|c|c|}
\hline \multirow{2}{*}{ Patient characteristics } & \multirow{2}{*}{$n$} & \multicolumn{2}{|c|}{ B7-H4 (ng/mL) } & \multicolumn{2}{|c|}{ OPN (ng/mL) } & \multicolumn{2}{|c|}{ TPS (U/L) } & \multicolumn{2}{|c|}{ CEA (ng/mL) } \\
\hline & & & $p$ & & $p$ & & $p$ & & $p$ \\
\hline \multicolumn{10}{|l|}{ Age } \\
\hline$<60$ & 32 & $97.64 \pm 15.64$ & \multirow{2}{*}{0.249} & $130.06 \pm 65.98$ & \multirow{2}{*}{0.516} & $96.25 \pm 17.59$ & \multirow{2}{*}{0.452} & $70.58 \pm 21.50$ & \multirow{2}{*}{0.764} \\
\hline$\geq 60$ & 27 & $93.57 \pm 10.06$ & & $111.60 \pm 47.57$ & & $101.74 \pm 36.28$ & & $85.03 \pm 13.54$ & \\
\hline \multicolumn{10}{|l|}{ Gender } \\
\hline Male & 41 & $94.38 \pm 12.24$ & \multirow{2}{*}{0.303} & $139.78 \pm 63.91$ & \multirow{2}{*}{0.373} & $100.01 \pm 33.14$ & \multirow{2}{*}{0.723} & $97.14 \pm 23.01$ & \multirow{2}{*}{0.279} \\
\hline Female & 18 & $98.13 \pm 15.23$ & & $113.68 \pm 48.70$ & & $96.66 \pm 14.75$ & & $76.51 \pm 43.64$ & \\
\hline \multicolumn{10}{|l|}{ Tumor masses } \\
\hline$<5 \mathrm{~cm}$ & 32 & $89.19 \pm 12.56$ & \multirow{2}{*}{0.016} & $110.87 \pm 41.61$ & \multirow{2}{*}{0.567} & $91.23 \pm 10.39$ & \multirow{2}{*}{0.027} & $63.24 \pm 39.87$ & \multirow{2}{*}{0.253} \\
\hline$\geq 5 \mathrm{~cm}$ & 27 & $102.68 \pm 45.29$ & & $113.82 \pm 57.77$ & & $109.19 \pm 30.17$ & & $92.71 \pm 53.42$ & \\
\hline \multicolumn{10}{|l|}{ Infiltration } \\
\hline Inside serosal layer & 35 & $86.75 \pm 11.31$ & \multirow{2}{*}{0.0001} & $100.47 \pm 42.68$ & \multirow{2}{*}{0.040} & $98.53 \pm 11.39$ & \multirow{2}{*}{0.962} & $56.94 \pm 59.83$ & \multirow{2}{*}{0.365} \\
\hline Reached and outside serosal layer & 24 & $100.41 \pm 12.10$ & & $145.21 \pm 60.06$ & & $98.89 \pm 33.16$ & & $92.61 \pm 34.21$ & \\
\hline \multicolumn{10}{|l|}{ Metastasized } \\
\hline Nonmetastasized & 48 & $83.34 \pm 25.23$ & \multirow{2}{*}{0.0041} & $121.50 \pm 28.26$ & \multirow{2}{*}{0.015} & $94.47 \pm 29.98$ & \multirow{2}{*}{0.021} & $61.00 \pm 33.23$ & \multirow{2}{*}{0.069} \\
\hline Metastasized & 11 & $116.34 \pm 40.82$ & & $110.68 \pm 57.50$ & & $123.01 \pm 44.55$ & & $95.00 \pm 39.82$ & \\
\hline Lymph node metastasis & & & & & & & & & \\
\hline$<4$ & 17 & $91.945 \pm 19.43$ & & $108.57 \pm 50.32$ & 0252 & $98.50 \pm 29.17$ & ( 412 & $74.96 \pm 26.85$ & 106 \\
\hline$\geq 4$ & 42 & $110.70 \pm 16.52$ & 0.0052 & $136.99 \pm 63.07$ & 0.353 & $105.15 \pm 10.50$ & 0.443 & $81.57 \pm 19.16$ & 0.106 \\
\hline Differentiation & & & & & & & & & \\
\hline Low & 7 & $91.72 \pm 9.62$ & & $113.47 \pm 44.02$ & & $98.30 \pm 16.91$ & & $90.24 \pm 17.66$ & \\
\hline Middle & 47 & $101.46 \pm 11.48$ & 0.135 & $120.43 \pm 29.01$ & 0.203 & $104.52 \pm 27.24$ & 0.573 & $82.47 \pm 23.09$ & 0.109 \\
\hline High & 5 & $100.42 \pm 17.34$ & & $121.06 \pm 31.33$ & & $102.84 \pm 15.22$ & & $85.24 \pm 32.6$ & \\
\hline Histology & & & & & & & & & \\
\hline Squamous & 2 & $98.72 \pm 11.56$ & 0283 & $122.47 \pm 35.16$ & 0121 & $103.88 \pm 12.46$ & 0332 & $85.58 \pm 31.50$ & 0238 \\
\hline Mucoid & 57 & $96.28 \pm 15.34$ & 0.203 & $117.29 \pm 40.91$ & 0.121 & $95.45 \pm 39.67$ & 0.532 & $75.42 \pm 33.54$ & 0.250 \\
\hline
\end{tabular}

node metastases $(91.945 \pm 19.43 \mathrm{ng} / \mathrm{mL})(P=0.0052)$, suggesting that $\mathrm{B} 7-\mathrm{H} 4$ was related to tumor progression and metastasis in CRC. We also evaluated the associations of serum B7-H4, OPN, TPS, and CEA levels with distant metastases in patients with CRC. The mean serum level of B7-H4 was significantly higher in patients with advanced tumor stage compared in those with early tumor stage $(P=0.017)$, and Mann-Whitney $U$ analysis also showed that B7-H4, OPN, and TPS serum expressions were significantly related to distant metastasis $(P=0.0041, P=0.015$, and $P=0.021$, respectively). However, there was no relationship between CEA levels $(P=0.069)$ and distant metastasis (Table 3).

3.4. Assessment of Preoperative and Postoperative Serum Markers. All 59 patients were evaluated preoperatively before radical resection for CRC. Twenty-one patients also underwent peripheral lymph node dissection. No patients experienced surgical or perioperative mortality, and there were no perioperative complications including hemorrhage and intestinal fistula. Serum expressions of B7-H4, OPN, TPS, and CEA detected again on the 7 th postoperative day had all decreased significantly compared with preoperative levels $(P=0.0064, P=0.041, P=0.016$, and $P=0.012$, respectively) (Table 5, Figure 3 ) and were similar to the levels in the healthy control group $(P<0.05)$. These results confirmed the relationships between these biomarkers and clinicopathologic features in CRC.

\section{Discussion}

China has experienced a two- to fourfold increase in the incidence of CRC during the past few decades [18, 19]. Although changes in dietary habits and lifestyle are believed to largely account for this increase, the interaction between environmental and genetic factors might play a pivotal role in colorectal carcinogenesis in Asian populations. Although early diagnosis and treatment have greatly improved the prognosis of this aggressive neoplasm [24,25], the almost complete lack of symptoms in the early stages and the absence of highly sensitive, effective, and economical methods of screening make it difficult to detect CRC before dissemination of the tumor. It would therefore be useful to identify individual or combined serum tumor markers that could aid in the early detection of CRC.

B7-H4 is a novel member of the inhibitory B7 family and is regarded as a negative regulator of the T-cell-mediated immune response and a potential diagnostic marker for CRC [17]. Previous studies demonstrated that B7-H4 was highly expressed in many different types of human cancers and was mostly associated with poor clinical outcomes [26]. 


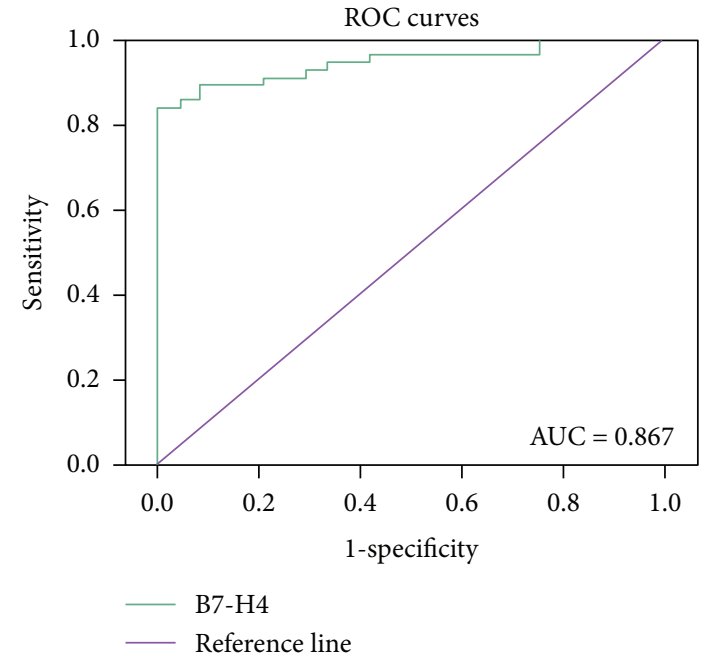

(a)

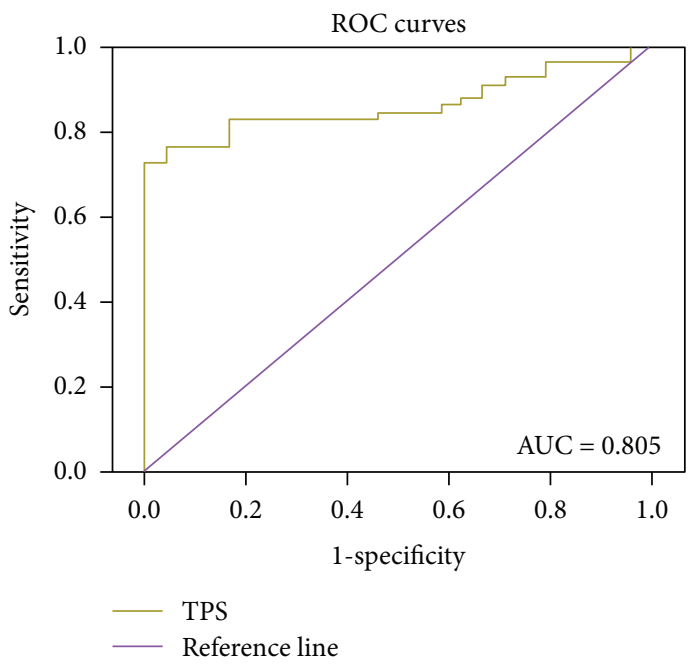

(c)

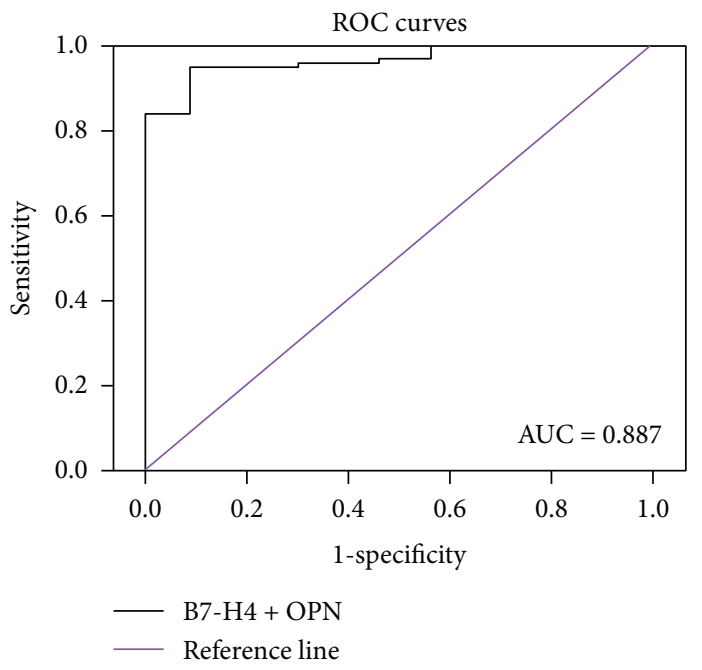

(e)

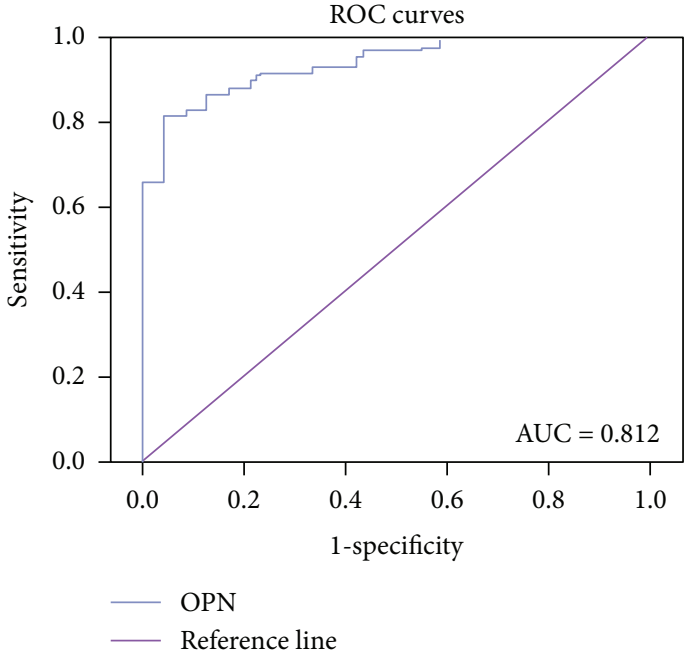

(b)

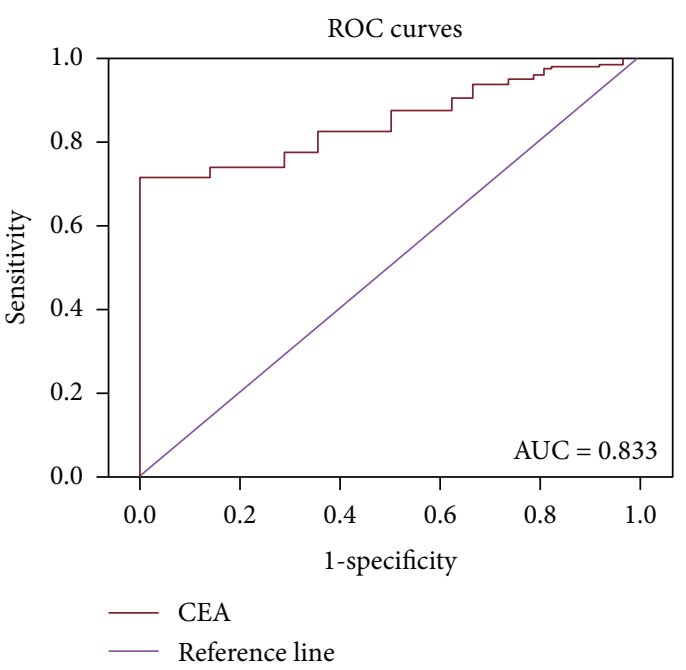

(d)

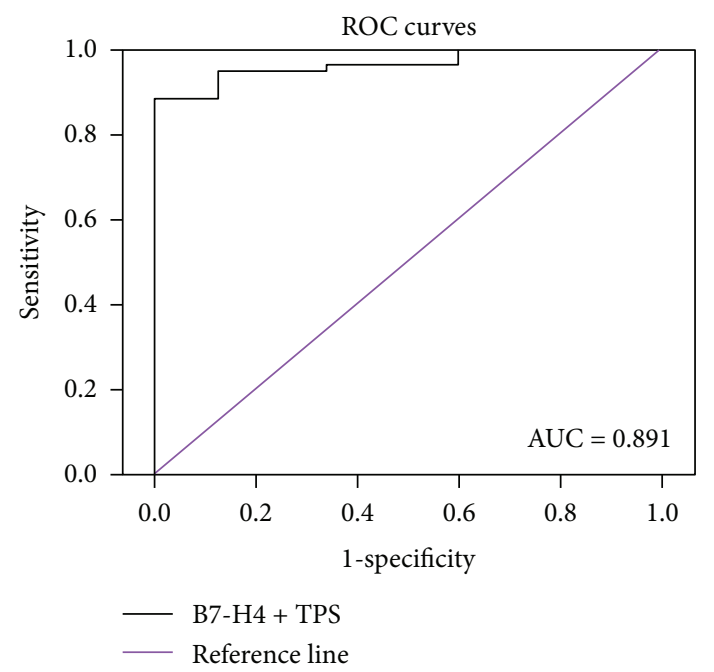

(f)

Figure 1: Continued. 


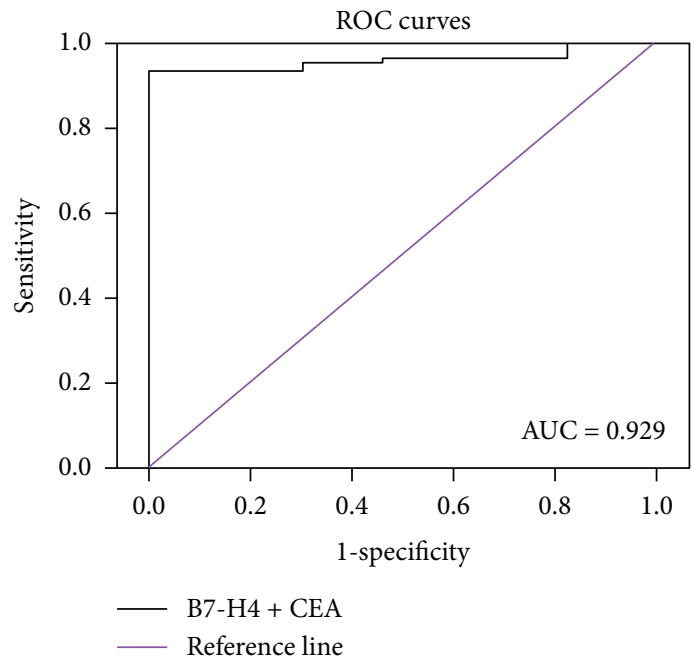

(g)

FIGURE 1: ROC curves of B7-H4/OPN/TPS/CEA of CRC patients. Receiver operating characteristic (ROC) curve analysis using four biomarkers to differentiate patients. Diagnostic accuracy of biomarkers was determined by obtaining the largest possible area under the curve (AUC) in ROC analysis. (a) B7-H4, (b) OPN, (c) TPS, (d) CEA, (e) B7-H4 + OPN, (f) B7-H4 + TPS, and (g) B7-H4 + CEA.

TABLE 4: The diagnosis value of serum B7-H4/OPN/TPS/CEA in CRC patients.

\begin{tabular}{lccc}
\hline Test variable & Sensitivity (\%) & Specificity (\%) & Youden index \\
\hline B7-H4 & 88.2 & 66.2 & 0.544 \\
OPN & 79.8 & 61.7 & 0.415 \\
TPS & 76.3 & 67.5 & 0.438 \\
CEA & 59.3 & 83.3 & 0.426 \\
Combinational & 99.2 & 55.9 & 0.511 \\
$\begin{array}{l}\text { (B7-H4 + OPN) } \\
\text { Combinational }\end{array}$ & 99.0 & 60.1 & 0.591 \\
$\begin{array}{l}\text { (B7-H4 + TPS) } \\
\text { Combinational }\end{array}$ & 98.9 & 80.4 & 0.793 \\
(B7-H4+CEA) & & & \\
\hline
\end{tabular}

B7-H4 was overexpressed in most CRC tumor tissues and was associated with infiltration depth and lymph node metastasis, which are associated with a poorer clinical outcome in patients with CRC [17, 27]. However, although serum B7-H4 levels have been considered to have diagnostic value in several human cancers, information on its use for the early diagnosis in CRC is currently lacking.

In the current study, we measured B7-H4 levels in CRC serum samples and showed that mean serum expression was significantly higher in CRC patients compared with healthy controls. These results were consistent with previous studies that reported increased B7-H4 expression in blood samples from patients with ovarian cancer, gastric cancer, and renal cell carcinoma $[28,29]$. Serum B7-H4 might thus serve as a potential biomarker of CRC. We further analyzed the correlations between serum B7-H4 and clinicopathologic features of CRC and showed that B7-H4 overexpression was closely associated with tumor size, lymph node metastasis, and tumor infiltration but not with gender or age. Using a

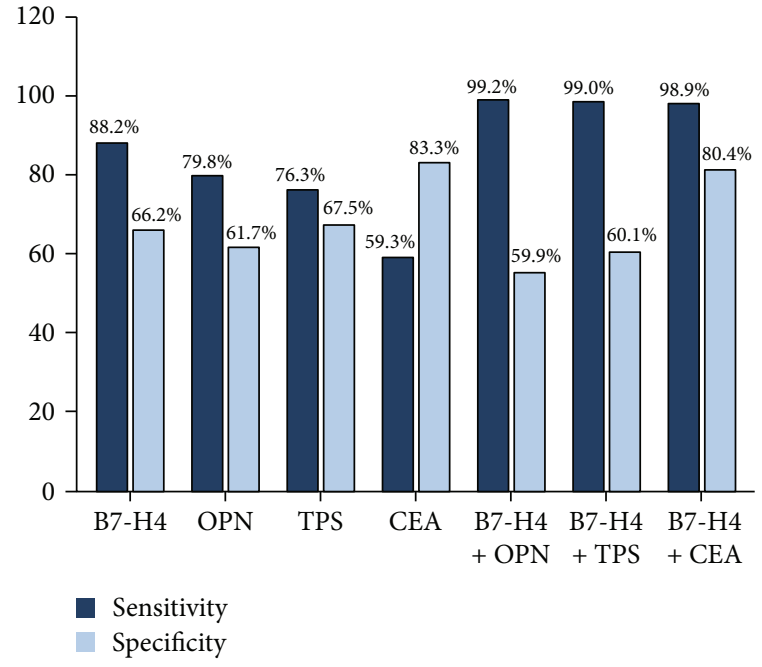

FIgure 2: The diagnosis value of serum B7-H4/OPN/TPS/CEA in CRC patients. Combined analysis using $\mathrm{B} 7-\mathrm{H} 4$ and CEA revealed sensitivity of $98.9 \%$ and specificity of $80.4 \%$ for discriminating CRC patients from healthy controls.

combination of different biomarkers has been reported to be a useful strategy [30-33] by increasing the sensitivity and specificity compared with the individual biomarkers alone. Although serum expressions of other tumor markers such as OPN, TPS, and CEA differed between CRC patients and healthy controls, they were not closely related to the clinicopathologic parameters of CRC, thus emphasizing the importance of $\mathrm{B} 7-\mathrm{H} 4$ in the development and progression of CRC. Moreover, higher serum expression of B7-H4 in CRC patients was also correlated with distant metastases, indicating that serum B7-H4 may be a potential diagnostic marker for distant metastases in CRC patients. 
TABLE 5: The comparison of serum concentrations of B7-H4/OPN/TPS/CEA between preoperative and postoperative groups in CRC patients.

\begin{tabular}{lccccc}
\hline Group & $n$ & B7-H4 $(\mathrm{ng} / \mathrm{mL})$ & OPN $(\mathrm{ng} / \mathrm{mL})$ & TPS $(\mathrm{U} / \mathrm{L})$ & CEA $(\mathrm{ng} / \mathrm{mL})$ \\
\hline Preoperative & 59 & $95.78 \pm 13.42$ & $116.14 \pm 56.31$ & $98.77 \pm 27.62$ & $77.17 \pm 81.09$ \\
Postoperative & 59 & $55.77 \pm 12.28$ & $78.38 \pm 35.59$ & $76.48 \pm 13.77$ & $30.52 \pm 95.30$ \\
$t$ & & 6.070 & 3.620 & 5.579 & 2.661 \\
$P$ & & 0.0064 & 0.041 & 0.016 & 0.012 \\
\hline
\end{tabular}

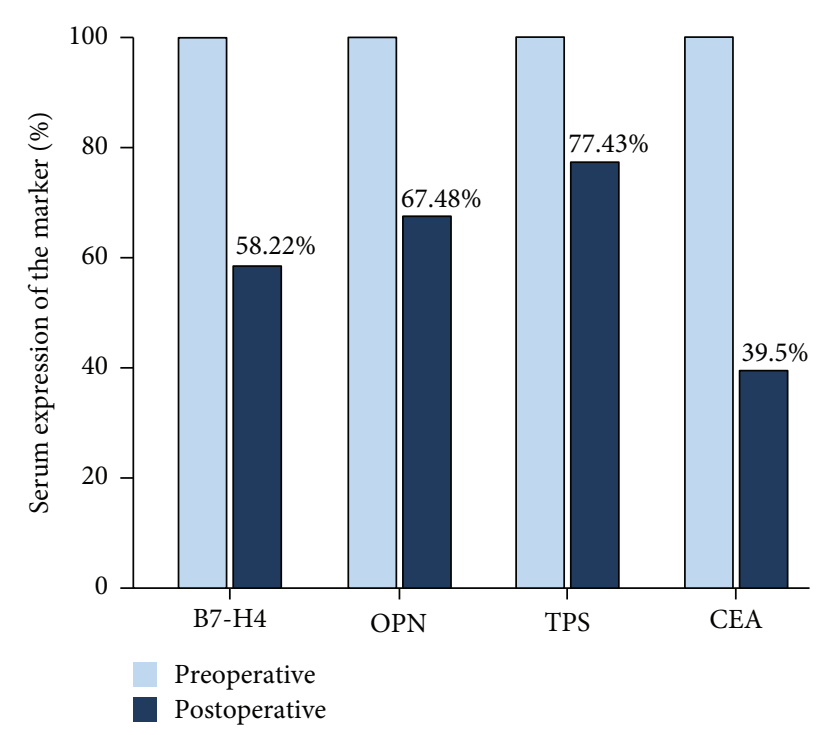

FIGURE 3: The comparison of serum concentrations of B7-H4/ OPN/TPS/CEA between preoperative and postoperative groups in CRC patients.

We further evaluated serum B7-H4 levels before and 7 days after radical resection of $\mathrm{CRC}$ and showed that $\mathrm{B} 7-\mathrm{H} 4$ levels decreased significantly during the first postoperative week, then recovered to normal. Given the positive correlation between $\mathrm{B} 7-\mathrm{H} 4$ protein expression in CRC tumor tissues [17], these results suggest that assessment of serum expression of B7-H4 after surgery might provide a tool for assessing the success and curative effect of surgery in CRC patients.

We also determined if the combined detection of B7-H4 with OPN, TPS, or CEA could increase the specificity and sensitivity of B7-H4 for CRC diagnosis. B7-H4 alone exhibited high sensitivity and low specificity, while CEA exhibited the opposite properties. OPN and TPS did not have high sensitivity or specificity for CRC diagnosis. We performed ROC analysis using the two markers combined and showed that the combination provided relatively high sensitivity and specificity for discriminating between CRC patients and healthy controls. No reliable independent biomarker has yet been established for CRC screening, and the current results suggest that the combination of $\mathrm{B} 7-\mathrm{H} 4$ and CEA serum assays may offer a useful tool for the detection of CRC.

In summary, elevated serum expression of B7-H4 may play a critical role in the development and progression of CRC. Detection of B7-H4 might thus serve as a clinical predictor of the diagnosis or outcomes in patients with CRC. The combined detection of B7-H4 and CEA significantly increased the sensitivity and specificity of CRC diagnosis compared to individual detection of these markers, suggesting that this combination may represent a potentially novel laboratory method for the clinical diagnosis of CRC. However, the sample size in the current study was relatively small. Further larger scale experiments and other disease cases that need to differentiate with CRC such as chronic enteritis need to confirm the enhanced diagnostic and prognostic values of combined detection of serum B7-H4 and CEA in CRC patients.

\section{Data Availability}

The data used to support the findings of this study are available from the corresponding author upon request.

\section{Conflicts of Interest}

The authors declare that they have no competing interests.

\section{Authors' Contributions}

Peng Wang and Chun Li performed the statistical analysis and prepared the manuscript. Xiaodong Gai was responsible for the study design and coordination, guided the statistical analysis, and revised the manuscript. Peng Wang and Xiaodong Gai were responsible for the study design and coordination and reviewed the manuscript critically. Peng Wang, Chun Li, Fang Zhang, and Xuzhe Ma collected the data and reviewed the manuscript. All authors read and approved the final manuscript.

\section{Acknowledgments}

This study was supported by the Science and Technology Department of Jilin province $(20170414030 \mathrm{GH})$, the Jilin Department of Health (2016J083, 2017J088), and the "Thirteenth Five-Year Plan" Science and Technology Research Projects of the Education Department of Jilin Province (JJKH20180353KJ, JJKH20180328KJ). We thank Susan Furness, PhD, from Liwen Bianji, Edanz Group China (http://www.liwenbianji.cn/ac), for editing the English text of a draft of this manuscript. 


\section{References}

[1] T. Norat, D. Aune, D. Chan, and D. Romaguera, "Fruits and vegetables: updating the epidemiologic evidence for the WCRF/AICR lifestyle recommendations for cancer prevention," Cancer Treatment and Research, vol. 159, no. 1, pp. 35-50, 2014.

[2] Z. Fang, C. Gong, S. Yu et al., "NFYB-induced high expression of E2F1 contributes to oxaliplatin resistance in colorectal cancer via the enhancement of CHK1 signaling," Cancer Letters, vol. 415, no. 2018, pp. 58-72, 2018.

[3] J. Smeby, A. Sveen, M. A. Merok et al., "CMS-dependent prognostic impact of KRAS and $B R A F^{\mathrm{V} 600 \mathrm{E}}$ mutations in primary colorectal cancer," Annals of Oncology, vol. 29, no. 5, pp. 1227-1234, 2018.

[4] K. W. Kinzler and B. Vogelstein, "Lessons from hereditary colorectal cancer," Cell, vol. 87, no. 2, pp. 159-170, 1996.

[5] M. H. Chew, W. S. Tan, Y. Liu, and P. Y. Cheah, "Genomics of hereditary colorectal cancer: lessons learnt from 25 years of the Singapore polyposis registry," Annals of the Academy of Medicine Singapore, vol. 44, no. 8, pp. 290-296, 2015.

[6] A. Chiaravalloti, A. Fiorentini, E. Palombo et al., "Evaluation of recurrent disease in the re-staging of colorectal cancer by 18-F-FDG PET/CT: use of CEA and CA 19-9 in patient selection," Oncology Letters, vol. 12, no. 5, pp. 4209-4213, 2016.

[7] C. O. Sahlmann, K. Homayounfar, M. Niessner et al., "Repeated adjuvant anti-CEA radioimmunotherapy after resection of colorectal liver metastases: safety, feasibility, and long-term efficacy results of a prospective phase 2 study," Cancer, vol. 123, no. 4, pp. 638-649, 2017.

[8] K. M. Yang, I. J. Park, C. W. Kim, S. A. Roh, D. H. Cho, and J. C. Kim, "The prognostic significance and treatment modality for elevated pre- and postoperative serum CEA in colorectal cancer patients," Annals of Surgical Treatment and Research, vol. 91, no. 4, pp. 165-171, 2016.

[9] S. Petrovic, G. D. Radosavljevic, J. Pantic, I. Jovanovic, N. Jankovic, and N. Arsenijevic, "Circulating and tissue galectin-1 and galectin-3 in colorectal carcinoma: association with clinicopathological parameters, serum CEA, IL-17 and IL23," Journal of the Balkan Union of Oncology, vol. 21, no. 4, pp. 941-949, 2016.

[10] C. A. Chambers, M. S. Kuhns, J. G. Egen, and J. P. Allison, "CTLA-4-mediated inhibition in regulation oft cell responses: mechanisms and manipulation in tumor immunotherapy," Annual Review of Immunology, vol. 19, no. 1, pp. 565-594, 2001.

[11] G. B. Park, H. Song, Y. S. Kim et al., "Cell cycle arrest induced by engagement of B7-H4 on Epstein-Barr virus-positive B-cell lymphoma cell lines," Immunology, vol. 128, no. 3, pp. 360368, 2009.

[12] G. L. Sica, I. H. Choi, G. Zhu et al., "B7-H4, a molecule of the B7 family, negatively regulates $\mathrm{T}$ cell immunity," Immunity, vol. 18, no. 6, pp. 849-861, 2003.

[13] W. Zou and L. Chen, "Inhibitory B7-family molecules in the tumour microenvironment," Nature Reviews Immunology, vol. 8, no. 6, pp. 467-477, 2008.

[14] P. Leandersson, G. Kalapotharakos, E. Henic et al., "A biomarker panel increases the diagnostic performance for epithelial ovarian cancer type I and II in young women," Anticancer Research, vol. 36, no. 3, pp. 957-965, 2016.

[15] E. R. Parra, P. Villalobos, J. Zhang et al., "Immunohistochemical and image analysis-based study shows that several immune checkpoints are co-expressed in non-small cell lung carcinoma tumors," Journal of Thoracic Oncology, vol. 13, no. 6, pp. 779-791, 2018.

[16] H. Huang, C. Li, and G. Ren, "Clinical significance of the B7-H4 as a novel prognostic marker in breast cancer," Gene, vol. 623, pp. 24-28, 2017.

[17] L. W. Zhao, C. Li, R. L. Zhang et al., "B7-H1 and B7-H4 expression in colorectal carcinoma: correlation with tumor FOXP $^{+}$regulatory T-cell infiltration," Acta Histochemica, vol. 116, no. 7, pp. 1163-1168, 2014.

[18] V. Barak, A. Meirovitz, V. Leibovici et al., "The diagnostic and prognostic value of tumor markers (CEA, SCC, CYFRA 21-1, TPS) in head and neck cancer patients," Anticancer Research, vol. 35, no. 10, pp. 5519-5524, 2015.

[19] W. Likui, W. Hong, Z. Shuwen, Y. Yuangang, and W. Yan, "The potential of osteopontin as a therapeutic target for human colorectal cancer," Journal of Gastrointestinal Surgery, vol. 15, no. 4, pp. 652-659, 2011.

[20] R. Partyka, A. Sandelewski, I. Łobejko, J. Kocot, P. Jałowiecki, and D. Kokocińska, "Usefulness of evaluation of soluble fragment cytokeratin 18, carcinoembryonic antigen and gastrointestinal carcinoma-associated antigen in diagnostic of patients with colorectal cancer," Polski Merkuriusz Lekarski, vol. 29 , no. 170 , pp. 128-130, 2010.

[21] R. Holmer, G. H. Wätzig, S. Tiwari, S. Rose-John, and H. Kalthoff, "Interleukin-6 trans-signaling increases the expression of carcinoembryonic antigen-related cell adhesion molecules 5 and 6 in colorectal cancer cells," BMC Cancer, vol. 15, no. 1, pp. 975-912, 2015.

[22] L. Ng, T. Wan, A. Chow et al., "Osteopontin overexpression induced tumor progression and chemoresistance to oxaliplatin through induction of stem-like properties in human colorectal cancer," Stem Cells International, vol. 2015, no. 2015, Article ID 247892, 8 pages, 2015.

[23] Ł. Pietrzyk, "Biomarkers discovery for colorectal cancer: a review on tumor endothelial markers as perspective candidates," Disease Markers, vol. 2016, no. 1, Article ID 4912405, 11 pages, 2016

[24] D. Yazilitas, N. Özdemir, O. Yazıc1, C. Hocazade, N. S. Demirci, and N. Zengin, "The clinical and pathological features affecting the time of relapse in patients with early stage colorectal cancer," Journal of Cancer Research and Therapeutics, vol. 12, no. 4, pp. 1257-1260, 2016.

[25] E. Gabrielli, A. J. Bastiampillai, M. Pontello et al., "Observational study to evaluate the impact of internet reminders for GPs on colorectal cancer screening uptake in Northern Italy in 2013," Journal of Preventive Medicine and Hygiene, vol. 57, no. 4, pp. 211-215, 2016.

[26] C. He, H. Qiao, H. Jiang, and X. Sun, “The inhibitory role of B7-H4 in antitumor immunity: association with cancer progression and survival," Clinical and Developmental Immunology, vol. 2011, article 695834, 8 pages, 2011.

[27] H.-x. Peng, W.-q. Wu, D.-m. Yang et al., "Role of B7-H4 siRNA in proliferation, migration, and invasion of LOVO colorectal carcinoma cell line," Biomed Research International, vol. 2015, no. 2015, Article ID 326981, 10 pages, 2015.

[28] H. Shi, M. Ji, J. Wu et al., "Serum B7-H4 expression is a significant prognostic indicator for patients with gastric cancer," World Journal of Surgical Oncology, vol. 12, no. 1, pp. 188185, 2014.

[29] R. H. Thompson, X. Zang, C. M. Lohse et al., "Serum-soluble $\mathrm{B} 7 \mathrm{x}$ is elevated in renal cell carcinoma patients and is 
associated with advanced stage," Cancer Research, vol. 68, no. 15, pp. 6054-6058, 2008.

[30] T. Schepeler, J. T. Reinert, M. S. Ostenfeld et al., "Diagnostic and prognostic microRNAs in stage II colon cancer," Cancer Research, vol. 68, no. 15, pp. 6416-6424, 2008.

[31] X. Chen, Y. Ba, L. Ma et al., "Characterization of microRNAs in serum: a novel class of biomarkers for diagnosis of cancer and other diseases," Cell Research, vol. 18, no. 10, pp. 9971006, 2008.

[32] Y. Chen, S. G. Gao, J. M. Chen et al., "Serum CA242, CA199, CA125, CEA, and TSGF are biomarkers for the efficacy and prognosis of cryoablation in pancreatic cancer patients," Cell Biochemistry and Biophysics, vol. 71, no. 3, pp. 1287-1291, 2015.

[33] Q. Wang, Z. Huang, S. Ni et al., "Plasma miR-601 and miR760 are novel biomarkers for the early detection of colorectal cancer," PLoS One, vol. 7, no. 9, article e44398, 2012. 


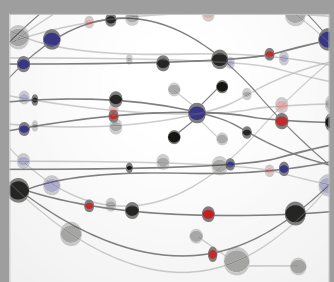

The Scientific World Journal
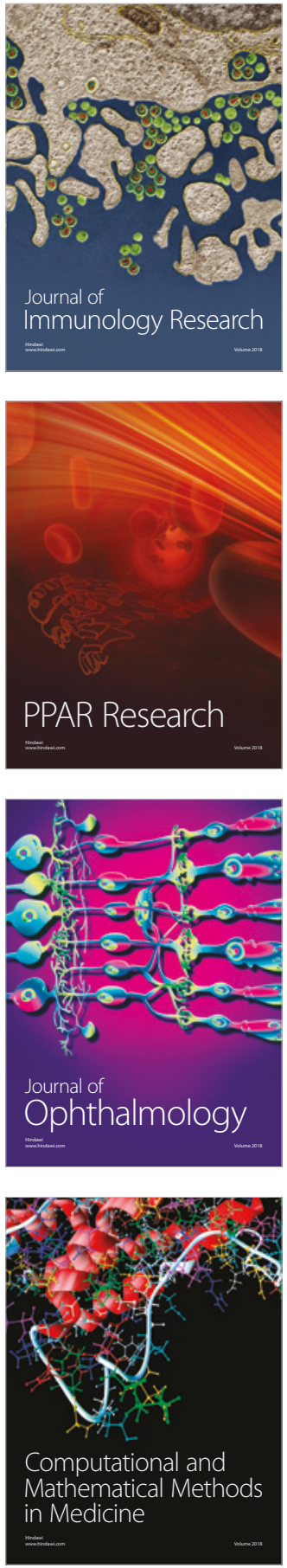

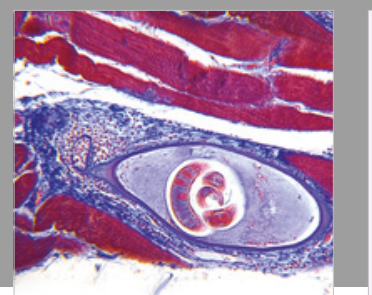

Gastroenterology Research and Practice

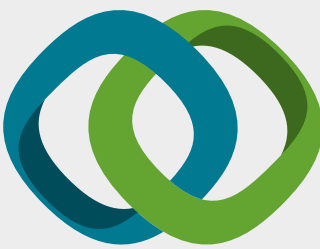

\section{Hindawi}

Submit your manuscripts at

www.hindawi.com
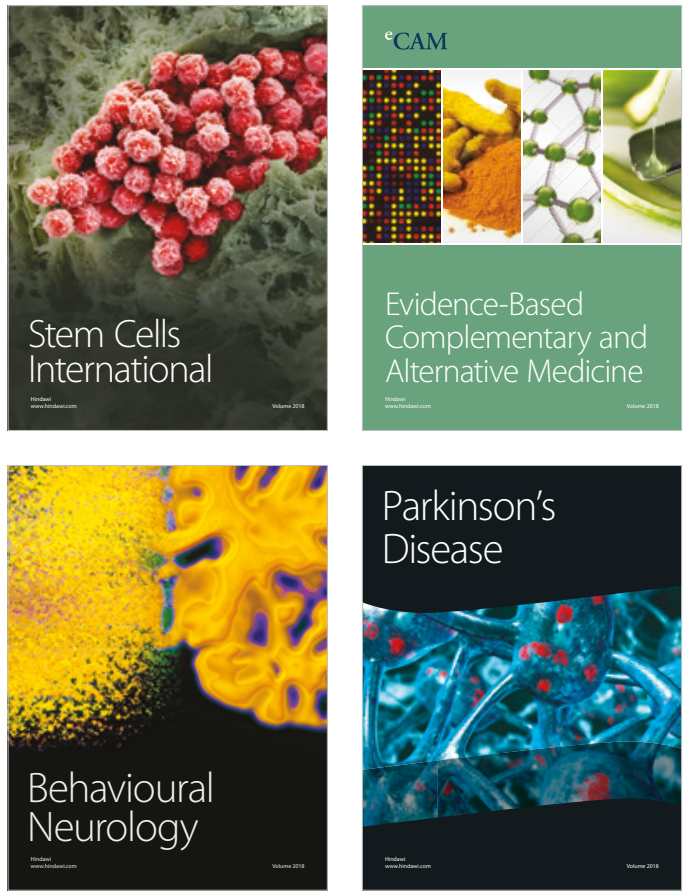

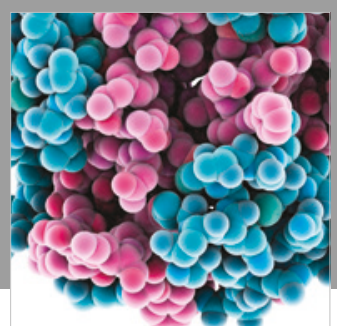

ournal of

Diabetes Research

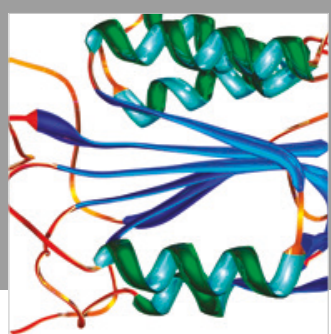

Disease Markers
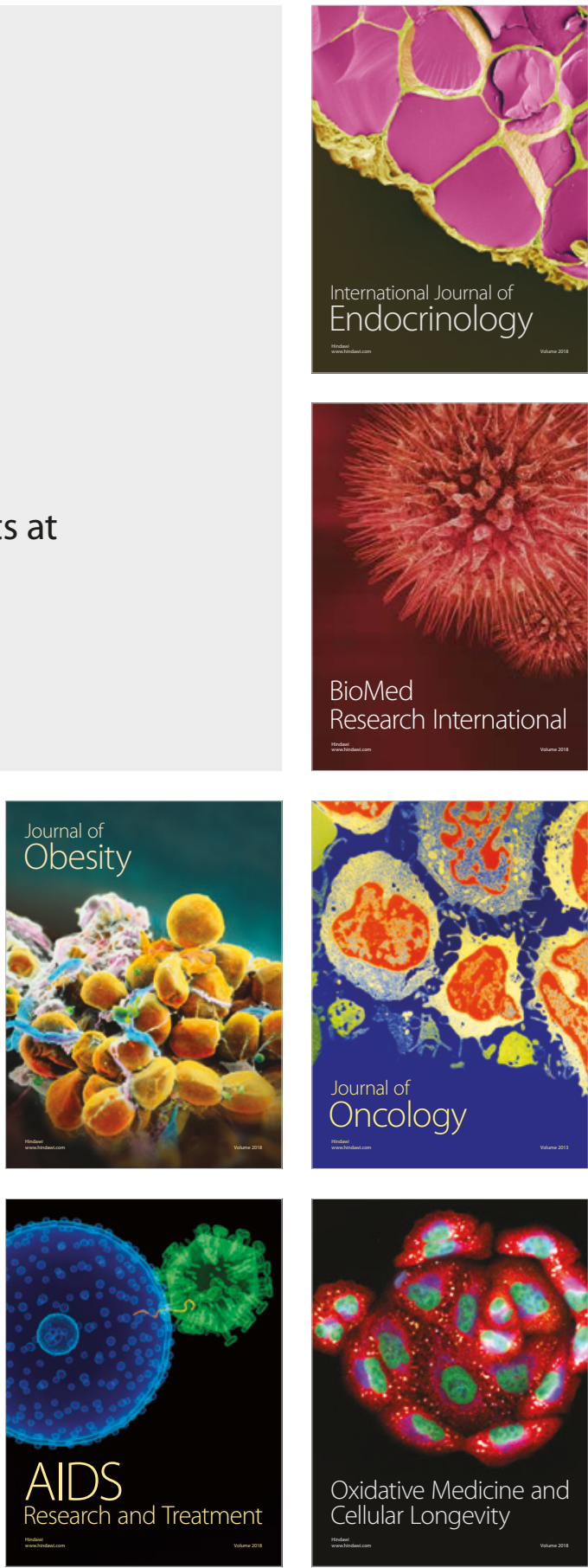